\title{
Efficacy of some native entomopathogenic nematodes against the alfalfa weevil, Hypera postica (Gyllenhal) (Coleoptera: Curculionidae), and the lucerne beetle, Gonioctena fornicata (Brüggemann) (Coleoptera: Chrysomelidae), adults under laboratory conditions
}

Ayşegül Çağlayan, Turgut Atay* (i) and IIlker Kepenekci

\begin{abstract}
Background: Entomopathogenic nematodes (EPNs) have more important role in biological control of economic insect pests. The effect of native EPNs on adults of the lucerne beetle, Gonioctena fornicata (Brüggemann, 1873) (Coleoptera: Chrysomelidae), and the alfalfa weevil, Hypera postica (Gyllenhal, 1813) (Coleoptera: Curculionidae), which are important alfalfa pests in Turkey and around the world, was investigated.

Results: Dose-mortality assays were carried out with 5 isolates [Steinernema carpocapsae (Weiser, 1955) (Nematoda: Steinernematidae) (Black sea isolate), S. feltiae Filipjev, 1934 (isolate 09-31), Heterorhabditis bacteriophora Poinar, 1976 (Nematoda: Heterorhabditidae) (isolate 09-43), H. bacteriophora Tokat-Songut, and S. carpocapsae Tokat-Ulas] using doses of 500, 1000, and $2000 \mathrm{IJs} \mathrm{ml}^{-1}$ under the laboratory conditions. Studies showed that all isolates had an effect $90 \%$ and more at $2000 \mathrm{Js} \mathrm{ml}^{-1}$ and at the end of $112 \mathrm{~h}$ [except, H. bacteriophora (isolate 09-43) and H. bacteriophora Tokat-Songut isolates against $H$. postica]. In addition, $\mathrm{LT}_{30}, \mathrm{LT}_{50}$, and $L T_{90}$ values at $1000 \mathrm{IJs} \mathrm{ml}^{-1}$ were determined.
\end{abstract}

Conclusions: According to the results, G. fornicata adults were susceptible to all isolates tested in the study and $H$. postica adults were susceptible to the isolates S. carpocapsae (Black sea isolate), S. feltiae (isolate 09-31), and S. carpocapsae Tokat-Ulas. This is the first study conducted in Turkey for the virulence of EPNs against G. fornicata and H. postica.

Keywords: Entomopathogenic nematodes, Virulence, Hypera postica, Gonioctena fornicata

\footnotetext{
*Correspondence: turgut.atay@gop.edu.tr

Department of Plant Protection, Faculty of Agriculture, Tokat Gaziosmanpasa University, Tokat, Turkey
}

\section{SpringerOpen}

(c) The Author(s). 2021 Open Access This article is licensed under a Creative Commons Attribution 4.0 International License, which permits use, sharing, adaptation, distribution and reproduction in any medium or format, as long as you give appropriate credit to the original author(s) and the source, provide a link to the Creative Commons licence, and indicate if changes were made. The images or other third party material in this article are included in the article's Creative Commons licence, unless indicated otherwise in a credit line to the material. If material is not included in the article's Creative Commons licence and your intended use is not permitted by statutory regulation or exceeds the permitted use, you will need to obtain permission directly from the copyright holder. To view a copy of this licence, visit http://creativecommons.org/licenses/by/4.0/. 


\section{Background}

Alfalfa [Medicago sativa L. (Fabaceae)] plant is a forage plant that provides a high-value feed and has a very dense production area in Turkey (Karakurt and Firıncioğlu 2003). The alfalfa weevil [Hypera postica (Gyllenhal, 1813) (Coleoptera: Curculionidae)] and the lucerne beetle [Gonioctena fornicata (Brüggemann, 1873) (Coleoptera: Chrysomelidae)] are among the alfalfa pests, spread to almost all grown areas of alfalfa in Turkey and cause significant crop losses if no control is made (Efe and Özgökçe 2014; Efil 2018). Larvae and adults of both species feed on leaves, young shoots, and the tips of the stems. As a result of feeding, the plant turns yellow and withers, and then the leaves dry completely (Atanasova and Semerdjieva 2009; Majić et al. 2013).

Frequent cuttings are the most effective cultural method of the pest in the alfalfa fields. Gözüaçık and İreç (2019) reported that cutting or grazing in the fall will reduce the spring larval population of alfalfa weevil. Chemical control against $H$. postica is recommended in Turkey, but no chemical pesticides have been registered for the control of G. fornicata. Therefore, pest control activities that are environmentally friendly are needed.

Entomopathogens nematodes (EPNs) are highly virulent. They have recently become one of the most emphasized groups due to these features. Studies have indicated that more than 30 nematode families are in contact with insects (Kaya and Stock 1997). EPNs have a variety of distinct and advantageous characteristics, such as high virulence and the ability to actively seek out hosts, making them promising chemical alternatives (Gulcu et al. 2017). EPNs are now used in many countries to control a wide range of economically important insect pests (Odendaal et al. 2015; Belien 2018). EPNs belong to the Steinernematidae and Heterorhabditidae families and live in the soil and are obligatory insect pathogens. They are one of the most frequently used groups in microbial control of pests (Glazer and Lewis 2000).

Studies using EPNs against G. fornicata and H. postica are limited (Falahi et al. 2011; Roodaki et al. 2011). Therefore, the aim of this study was to investigate the virulence of EPNs against G. fornicata and $H$. postica adults under laboratory conditions.

\section{Methods}

\section{Nematode sources}

Native Turkish EPNs, Steinernema feltiae (isolate 0931) from a vegetable garden in Aydin, S. carpocapsae (Black sea isolate) from grassland in Rize, and Heterorhabditis bacteriophora (isolate 09-43) from peach orchard in Aydın, Turkey, were obtained and supplied by Prof. Dr. Selçuk HAZIR (Aydın Adnan Menderes University, Faculty of Arts and Sciences, Department of Biology, Aydin, Turkey). S. carpocapsae Tokat-Ulas and $H$. bacteriophora Tokat-Songut were isolated from alfalfa cultivated areas of Tokat province (Turkey) (Çağlayan et al. 2020). The nematodes were cultured in last instar larvae of wax moth, Galleria mellonella (Linnaeus, 1758) (Lepidoptera: Pyralidae) at room temperature $\left(23-24,{ }^{\circ} \mathrm{C}\right)$ using methods described by Kaya and Stock (1997). The harvested infective juveniles (IJs) were used within 2 weeks after emergence for the experiments.

\section{Insect sources}

The lucerne beetle and alfalfa weevil adults were obtained from alfalfa fields in Emirseyit town of Tokat, Turkey $\left(40^{\circ} 2^{\prime} 13^{\prime \prime} \mathrm{N}, 36^{\circ} 23^{\prime} 59^{\prime \prime} \mathrm{E}, 554 \mathrm{~m}\right)$.

\section{Bioassays}

Experiments were carried out in Petri-dishes $(9 \mathrm{~cm}$ diameter). In the bottom of each Petri dish, filter paper was placed and $1 \mathrm{ml}$ of prepared EPNs concentrations was poured onto the filter paper. For the control group, $1 \mathrm{ml}$ of no-nematode water was used (Shahina and Salma 2010). In each Petri dish, 5 adult insects were released using the camel's hair brush. Each EPN species was applied at 3 concentrations 500,1000 , and $2000 \mathrm{IJs} \mathrm{ml}{ }^{-1}$. A micropipette was used to make the applications. After each treatment, the pipette tips were replaced. Alfalfa leaves were placed in Petri dishes to feed the insects. Experiments were carried at 4 replicates and 2 different times for each concentration. The data for mortality were recorded at 8-h intervals and the counts continued until to 120th hour. Dead insects were either dissected under a stereomicroscope or placed into White traps for nematode emergence to ensure that they were killed with EPNs (White 1927).

\section{Statistical analysis}

Data was analyzed using analysis of variance (ANOVA), and the means were compared by Tukey's multiple comparison test. All statistical analyses were carried out using the MINITAB Release 16 packet program. $\mathrm{LT}_{30}$, $\mathrm{LT}_{50}$, and $\mathrm{LT}_{90}$ values were calculated by using the Probit analysis.

\section{Results}

Mortality rates caused by EPNs isolates varied according to the test insect, time, isolates, and concentration. There was a positive correlation between nematode concentration and exposure time and mortality. S. carpocapsae (Black sea isolate) started to show a significant effect at all doses against G. 
fornicata from the 32nd hour (F:21.67, DF: 3.28, $P<$ 0.05 ), and this effect increased over $90 \%$ at the 64th hour (F:103.29, DF: 3.28, $P<0.05$ ) (Table 1). S. feltiae (isolate 09-31) was 92\% effective in $64 \mathrm{~h}$ at a dose of $2000 \mathrm{IJs} \mathrm{ml}^{-1}$. Other doses caused a mortality rate of over $50 \%$ at the same time period (F:30.51, DF: 3.27, $P<0.05)$. By the 112th hour, all doses produced over 90\% death (F:68.59, DF: 3.27, $P<0.05$ ) (Table 1). Similar to the $S$. carpocapsae (Black sea isolate) isolate, $S$. carpocapsae Tokat-Ulas showed a significant efficacy after $48 \mathrm{~h}$ and caused more than 90\% mortality at all doses (F:148.74, DF: 3.26, $P<$ 0.05) (Table 1). H. bacteriophora (isolate 09-43) caused an effect of $98 \%$ at the 120th hour at a dose of $2000 \mathrm{IJs} \mathrm{ml}^{-1}$, while this effect did not exceed $70 \%$ at other doses (F:34.25, DF: 3.21, $P<0.05$ ) (Table 2). $H$. bacteriophora Tokat-Songut revealed a mortality rate of over $90 \%$ at doses of 1000 and $2000 \mathrm{IJs} \mathrm{m}^{-1}$ at $72 \mathrm{~h}$ (F:54.81, DF: 3.28, $P<0.05$ ). At the 112th hour, the effect of all doses was over 90\% (F:91.73, DF: $3.28, P<0.05)$ (Table 2).

When the $\mathrm{LT}_{30}$ rates of the isolates against $G$. fornicata were examined, the most effective isolate was $S$. carpocapsae Tokat-Ulas. This was followed by S. carpocapsae (Black sea isolate), H. bacteriophora Tokat-Songut, S. feltiae (isolate 09-31), and $H$. bacteriophora (isolate 09-43). The order formed by the isolates for $\mathrm{LT}_{30}$ value is valid for $\mathrm{LT}_{50}$ and $\mathrm{LT}_{90}$ values (Table 3 ).

Steinernema carpocapsae (Black sea isolate) showed a mortality rate of over $90 \%$ against $H$. postica from 72 h onwards at all doses (F:57.55, DF: 3.26, $P<0.05$ ) (Table 4). Similarly, S. carpocapsae Tokat-Ulas caused a significant mortality rate against $H$. postica by more

Table 1 Mortality rates of Gonioctena fornicata adults exposed to Steinernema carpocapsae (Black sea isolate), S. feltiae (isolate 09-31), and S. carpocapsae Tokat-Ulas and controls over $120 \mathrm{~h}$ from treatment

\begin{tabular}{|c|c|c|c|c|c|c|c|c|}
\hline \multicolumn{9}{|c|}{ Mortality \pm SEM*(\%) } \\
\hline \multirow[t]{2}{*}{ HAT $^{* *}$} & \multicolumn{4}{|c|}{ Steinernema carpocapsae (Black sea isolate) } & \multicolumn{4}{|c|}{ S. feltiae (isolate 09-31) } \\
\hline & $500 \mathrm{IJs}$ & 1000 & 2000 & Control & $500 \mathrm{IJs}$ & 1000 & 2000 & Control \\
\hline 32 & $26.6 \pm 2.4 b^{* * *}$ & $50.3 \pm 4.7 \mathrm{ab}$ & $60.5 \pm 0.9 a$ & $0.0 \pm 0.0 c$ & $15.0 \pm 1.1 a^{1}$ & $15.4 \pm 2.5 \mathrm{a}$ & $33.7 \pm 3.20 \mathrm{a}$ & $0.0 \pm 0.0 b$ \\
\hline 40 & $55.3 \pm 6.5 b$ & $68.7 \pm 3.7 \mathrm{ab}$ & $92.1 \pm 3.8 \mathrm{a}$ & $0.0 \pm 0.0 c$ & $30.4 \pm 2.8 a$ & $31.4 \pm 3.7 a$ & $46.7 \pm 3.76 a$ & $0.0 \pm 0.0 b$ \\
\hline 48 & $85.0 \pm 4.3 b$ & $88.6 \pm 3.1 b$ & $100.0 \pm 0.0 a$ & $0.0 \pm 0.0 c$ & $35.8 \pm 3.6 a$ & $53.3 \pm 4.6 a$ & $60.2 \pm 1.38 a$ & $0.0 \pm 0.0 b$ \\
\hline 56 & $85.0 \pm 4.3 b$ & $92.0 \pm 3.5 \mathrm{ab}$ & $100.0 \pm 0.0 a$ & $0.0 \pm 0.0 c$ & $45.4 \pm 2.0 a$ & $61.0 \pm 3.8 a$ & $76.1 \pm 4.69 a$ & $0.0 \pm 0.0 b$ \\
\hline 64 & $90.3 \pm 2.6 b$ & $96.0 \pm 2.8 \mathrm{ab}$ & $100.0 \pm 0.0 a$ & $0.0 \pm 0.0 c$ & $51.4 \pm 1.5 b$ & $76.7 \pm 6.0 \mathrm{ab}$ & $92.0 \pm 3.46 a$ & $0.0 \pm 0.0 c$ \\
\hline 72 & $93.4 \pm 2.8 b$ & $97.0 \pm 2.0 a b$ & $100.0 \pm 0.0 a$ & $0.0 \pm 0.0 c$ & $57.7 \pm 2.3 b$ & $83.1 \pm 5.0 a b$ & $94.7 \pm 2.13 a$ & $0.0 \pm 0.0 c$ \\
\hline 80 & $98.0 \pm 2.6 a$ & $97.0 \pm 2.0 a$ & $100.0 \pm 0.0 a$ & $0.0 \pm 0.0 b$ & $57.7 \pm 2.3 b$ & $83.1 \pm 5.0 a b$ & $94.7 \pm 2.13 a$ & $0.0 \pm 0.0 c$ \\
\hline 88 & $98.0 \pm 2.6 a$ & $97.0 \pm 2.0 a$ & $100.0 \pm 0.0 a$ & $0.0 \pm 0.0 b$ & $57.7 \pm 2.3 b$ & $83.1 \pm 5.0 \mathrm{ab}$ & $94.7 \pm 2.13 a$ & $0.0 \pm 0.0 c$ \\
\hline 96 & $98.0 \pm 2.6 a$ & $99.7 \pm 0.9 a$ & $100.0 \pm 0.0 \mathrm{a}$ & $0.0 \pm 0.0 b$ & $77.9 \pm 2.5 a$ & $85.2 \pm 4.6 a$ & $94.7 \pm 2.13 a$ & $0.0 \pm 0.0 b$ \\
\hline 104 & $98.0 \pm 2.6 a$ & $99.7 \pm 0.9 a$ & $100.0 \pm 0.0 a$ & $0.0 \pm 0.0 b$ & $82.7 \pm 1.5 a$ & $88.6 \pm 3.1 a$ & $94.7 \pm 2.13 a$ & $0.0 \pm 0.0 b$ \\
\hline 112 & $98.0 \pm 2.6 a$ & $99.7 \pm 0.9 a$ & $100.0 \pm 0.0 a$ & $0.0 \pm 0.0 b$ & $91.5 \pm 2.9 a$ & $94.7 \pm 2.1 a$ & $94.7 \pm 2.13 a$ & $0.0 \pm 0.0 b$ \\
\hline \multirow[t]{3}{*}{120} & $99.3 \pm 2.0 a$ & $99.7 \pm 0.9 a$ & $100.0 \pm 0.0 a$ & $0.0 \pm 0.0 b$ & $91.5 \pm 2.9 a$ & $97.0 \pm 2.0 a$ & $97.0 \pm 2.00 a$ & $0.0 \pm 0.0 b$ \\
\hline & \multicolumn{8}{|c|}{ S. carpocapsae Tokat-Ulas } \\
\hline & $500 \mathrm{IJs}$ & & 1000 & & 2000 & & Control & \\
\hline 32 & $57.0 \pm 0.9 b$ & & $66.1 \pm 2.8 \mathrm{ab}$ & & $88.6 \pm 3.1 a$ & & $0.0 \pm 0.0 c$ & \\
\hline 40 & $85.8 \pm 1.3 a$ & & $84.6 \pm 2.5 a$ & & $97.0 \pm 2.0 \mathrm{a}$ & & $0.0 \pm 0.0 b$ & \\
\hline 48 & $94.7 \pm 2.2 \mathrm{a}$ & & $97.0 \pm 2.0 a$ & & $100.0 \pm 0.0 a$ & & $0.0 \pm 0.0 b$ & \\
\hline 56 & $97.6 \pm 2.0 a$ & & $97.0 \pm 2.0 a$ & & $100.0 \pm 0.0 a$ & & $0.0 \pm 0.0 b$ & \\
\hline 64 & $99.4 \pm 1.3 a$ & & $98.7 \pm 1.6 a$ & & $100.0 \pm 0.0 a$ & & $0.0 \pm 0.0 b$ & \\
\hline 72 & $99.4 \pm 1.3 a$ & & $98.7 \pm 1.6 a$ & & $100.0 \pm 0.0 a$ & & $0.0 \pm 0.0 b$ & \\
\hline 80 & $99.4 \pm 1.3 a$ & & $98.7 \pm 1.6 a$ & & $100.0 \pm 0.0 a$ & & $0.0 \pm 0.0 b$ & \\
\hline 88 & $100.0 \pm 0.0 a$ & & $100.0 \pm 0.0 a$ & & $100.0 \pm 0.0 a$ & & $0.0 \pm 0.0 b$ & \\
\hline 120 & $100.0 \pm 0.0 a$ & & $100.0 \pm 0.0 a$ & & $100.0 \pm 0.0 a$ & & $0.0 \pm 0.0 b$ & \\
\hline
\end{tabular}

*SEM: standard error of the mean

**HAT: hours after treatment

${ }^{* * *}$ Means in a line followed by the same letter are not statistical significantly different (ANOVA $P<0.05$, Tukey's test) 
Table 2 Mortality rates of Gonioctena fornicata adults exposed to Heterorhabditis bacteriophora (isolate 09-43) and H. bacteriophora Tokat-Songut and controls over $120 \mathrm{~h}$ from treatment

\begin{tabular}{|c|c|c|c|c|c|c|c|c|}
\hline \multicolumn{9}{|c|}{ Mortality \pm SEM*(\%) } \\
\hline \multirow[t]{2}{*}{$\mathrm{HAT}^{* *}$} & \multicolumn{4}{|c|}{ Heterorhabditis bacteriophora (isolate 09-43) } & \multicolumn{4}{|c|}{ H. bacteriophora Tokat-Songut } \\
\hline & $500 \mathrm{IJs}$ & 1000 & 2000 & Control & $500 \mathrm{IJs}$ & 1000 & 2000 & Control \\
\hline 32 & $0.0 \pm 0.0 a^{* * *}$ & $0.0 \pm 0.0 \mathrm{a}$ & $2.4 \pm 2.0 \mathrm{a}$ & $0.0 \pm 0.0 \mathrm{a}$ & $5.3 \pm 2.1 a b^{1}$ & $8.2 \pm 2.0 \mathrm{ab}$ & $16.9 \pm 5.0 \mathrm{a}$ & $0.0 \pm 0.0 \mathrm{~b}$ \\
\hline 40 & $0.9 \pm 1.5 \mathrm{a}$ & $0.6 \pm 1.3 \mathrm{a}$ & $9.0 \pm 4.0 \mathrm{a}$ & $0.0 \pm 0.0 \mathrm{a}$ & $34.2 \pm 2.2 \mathrm{a}$ & $33.9 \pm 3.7 a$ & $58.3 \pm 1.5 \mathrm{a}$ & $0.0 \pm 0.0 \mathrm{~b}$ \\
\hline 48 & $0.9 \pm 1.5 \mathrm{ab}$ & $7.0 \pm 3.2 \mathrm{ab}$ & $16.4 \pm 4.4 a$ & $0.0 \pm 0.0 \mathrm{~b}$ & $47.2 \pm 1.8 \mathrm{a}$ & $78.4 \pm 9.0 \mathrm{a}$ & $80.6 \pm 3.5 a$ & $0.0 \pm 0.0 \mathrm{~b}$ \\
\hline 56 & $0.9 \pm 1.5 \mathrm{ab}$ & $13.7 \pm 3.9 a b$ & $16.4 \pm 4.4 a$ & $0.0 \pm 0.0 \mathrm{~b}$ & $52.8 \pm 1.8 \mathrm{a}$ & $83.0 \pm 5.5 a$ & $86.9 \pm 3.9 a$ & $0.0 \pm 0.0 \mathrm{~b}$ \\
\hline 64 & $0.9 \pm 1.5 \mathrm{ab}$ & $19.2 \pm 5.9 a$ & $25.6 \pm 3.2 \mathrm{a}$ & $0.0 \pm 0.0 b$ & $63.4 \pm 1.5 b$ & $85.0 \pm 5.2 \mathrm{ab}$ & $94.8 \pm 3.6 a$ & $0.0 \pm 0.0 \mathrm{c}$ \\
\hline 72 & $12.6 \pm 4.7 \mathrm{ab}$ & $35.5 \pm 6.1 a$ & $50.0 \pm 9.4 a$ & $0.0 \pm 0.0 \mathrm{~b}$ & $68.9 \pm 3.3 b$ & $94.7 \pm 2.1 \mathrm{a}$ & $96.0 \pm 2.8 \mathrm{a}$ & $0.0 \pm 0.0 c$ \\
\hline 80 & $26.4 \pm 4.6 a$ & $46.7 \pm 2.9 a$ & $72.0 \pm 7.5 a$ & $0.0 \pm 0.0 b$ & $71.2 \pm 2.9 b$ & $94.7 \pm 2.1 \mathrm{a}$ & $96.0 \pm 2.8 \mathrm{a}$ & $0.0 \pm 0.0 c$ \\
\hline 88 & $30.4 \pm 6.1 a$ & $46.7 \pm 2.9 a$ & $78.1 \pm 5.6 a$ & $0.0 \pm 0.0 \mathrm{~b}$ & $73.7 \pm 2.8 b$ & $98.7 \pm 1.6 \mathrm{a}$ & $97.0 \pm 2.0 \mathrm{a}$ & $0.0 \pm 0.0 \mathrm{c}$ \\
\hline 96 & $52.4 \pm 1.2 \mathrm{a}$ & $46.7 \pm 2.9 a$ & $81.0 \pm 5.3 a$ & $0.0 \pm 0.0 \mathrm{~b}$ & $75.8 \pm 2.3 b$ & $98.7 \pm 1.6 \mathrm{a}$ & $97.0 \pm 2.0 \mathrm{a}$ & $0.0 \pm 0.0 c$ \\
\hline 104 & $60.4 \pm 0.8 a$ & $57.7 \pm 6.1 \mathrm{a}$ & $93.3 \pm 5.6 a$ & $0.0 \pm 0.0 \mathrm{~b}$ & $82.8 \pm 3.2 b$ & $98.7 \pm 1.6 \mathrm{a}$ & $97.0 \pm 2.0 \mathrm{ab}$ & $0.0 \pm 0.0 \mathrm{c}$ \\
\hline 112 & $64.7 \pm 1.1 \mathrm{a}$ & $68.7 \pm 7.5 \mathrm{a}$ & $93.3 \pm 5.6 a$ & $0.0 \pm 0.0 b$ & $90.6 \pm 4.4 a$ & $99.7 \pm 0.9 a$ & $99.7 \pm 0.9 a$ & $0.0 \pm 0.0 \mathrm{~b}$ \\
\hline 120 & $64.7 \pm 1.1 b$ & $68.7 \pm 7.5 b$ & $97.6 \pm 2.0 \mathrm{a}$ & $0.0 \pm 0.0 c$ & $90.6 \pm 4.4 b$ & $99.7 \pm 0.9 a b$ & $100.0 \pm 0.0 \mathrm{a}$ & $0.0 \pm 0.0 c$ \\
\hline
\end{tabular}

*SEM: standard error of the mean

**HAT: hours after treatment

***Means in a line followed by the same letter are not statistical significantly different (ANOVA $P<0.05$, Tukey's test)

than $85 \%$ from the 64th hour at all doses (F:31.87, DF: 3.27, $P<0.05$ ) (Table 4). S. feltiae (isolate 09-31) reached $90 \%$ effect only at $2000 \mathrm{IJs} \mathrm{ml}^{-1}$ doses and at the end of 112th hour (F:27.80, DF: 3.26, $P<0.05$ ) (Table 4). The rate of effect of $H$. bacteriophora (isolate 09-43) remained quite low than other isolates and did not exceed $26 \%$ at all doses at the end of 120th hour (F:8.41, DF: 3.24, $P<0.05$ ) (Table 5). At $120 \mathrm{~h}$, the effect of $H$. bacteriophora Tokat-Songut was only $37 \%$ at the highest dose and its effect remained quite low, similar to that of $H$. bacteriophora (isolate 09-43) (F:9.28, DF: 3.25, $P<0.05$ ) (Table 5).

When the LT rates of the isolates against $H$. postica were examined, the order formed by $\mathrm{LT}_{30}$ and $\mathrm{LT}_{50}$ values was $S$. carpocapsae (Black sea isolate), S. carpocapsae Tokat-Ulas, S. feltiae (isolate 09-31), H. bacteriophora Tokat-Songut, and H. bacteriophora (isolate 09-43). This ranking was $S$. carpocapsae Tokat-Ulas, S. carpocapsae
(Black sea isolate), S. feltiae (isolate 09-31), H. bacteriophora Tokat-Songut, and H. bacteriophora (isolate 09-43) at $\mathrm{LT}_{90}$ value (Table 6).

\section{Discussion}

The results showed that $S$. carpocapsae (Black sea isolate) and $S$. carpocapsae Tokat-Ulas were more effective against both $H$. postica and G. fornicata adults than other isolates. Similarly, Atay and Kepenekci (2016) reported that the $S$. carpocapsae (Black sea isolate) had an effect of 80,83 , and $82 \%$ on another alfalfa pest, Holotrichapion pullum (Gyllenhal, 1833) (Coleoptera: Apionidae) adults, at 20 degrees and at 3 different concentrations $\left(500,1000,5000 \mathrm{IJs} \mathrm{ml}^{-1}\right)$, respectively. In addition, Kepenekci et al. (2018) stated that $S$. carpocapsae (Black sea isolate) showed $99 \%$ efficacy in adults of Xylosandrus germanus (Blandford) (Coleoptera: Curculionidae) at a dose of $1000 \mathrm{IJs} \mathrm{m}^{-1}$. Brivio and Mastore (2018) emphasized that symbiotic

Table 3 Lethal time $\left(\mathrm{LT}_{30}, \mathrm{LT}_{50}\right.$, and $\left.\mathrm{LT}_{90}\right)$ values of treated isolates against adults of Gonioctena fornicata (hour)

\begin{tabular}{|c|c|c|c|c|c|}
\hline Isolates & Slope \pm SE & $\mathrm{LT}_{30}$ (95\% fiducial limit) & $\mathrm{LT}_{50}$ (95\% fiducial limit) & $\mathrm{LT}_{90}(95 \%$ fiducial limit) & Heterogeneity \\
\hline $\begin{array}{l}\text { Steinernema carpocapsae } \\
\text { (Black sea isolate) }\end{array}$ & $5.024 \pm 0.376$ & $29.584(25.789-32.900)$ & $37.621(33.943-41.041)$ & $67.688(61.707-75.841)$ & 1.39 \\
\hline S. feltiae (isolate 09-31) & $4.060 \pm 0.318$ & 40.000 (35.517-43.952) & $53.853(49.528-58.083)$ & $111.391(100.005-128.141)$ & 1.12 \\
\hline $\begin{array}{l}\text { Heterorhabditis bacteriophora } \\
\text { (isolate 09-43) }\end{array}$ & $4.181 \pm 0.383$ & $58.684(51.365-64.913)$ & 78.331 (71.189-86.845) & $158.650(133.011-210.174)$ & 1.96 \\
\hline H. bacteriophora Tokat-Songut & $5.729 \pm 0.424$ & $37.263(33.590-40.478)$ & $46.006(42.547-49.264)$ & $77.002(71.236-84.735)$ & 1.21 \\
\hline S. carpocapsae Tokat-Ulas & $5.999 \pm 0.502$ & $26.030(23.358-28.372)$ & $31.833(29.295-34.212)$ & $52.060(48.186-57.158)$ & 0.91 \\
\hline
\end{tabular}


Table 4 Mortality rates of Hypera postica adults exposed to Steinernema carpocapsae (Black sea isolate), S. feltiae (isolate 09-31), and S. carpocapsae Tokat-Ulas and controls over $120 \mathrm{~h}$ from treatment

\begin{tabular}{|c|c|c|c|c|c|c|c|c|}
\hline \multicolumn{9}{|c|}{ Mortality \pm SEM*(\%) } \\
\hline \multirow[t]{2}{*}{$\mathrm{HAT}^{* *}$} & \multicolumn{4}{|c|}{ Steinernema carpocapsae (Black sea isolate) } & \multicolumn{4}{|c|}{ S. feltiae (isolate 09-31) } \\
\hline & $500 \mathrm{IJs}$ & 1000 & 2000 & Control & $500 \mathrm{IJs}$ & 1000 & 2000 & Control \\
\hline 32 & $1.3 \pm 1.6 b^{* * *}$ & $31.1 \pm 3.3 \mathrm{a}$ & $54.3 \pm 2.5 a$ & $0.0 \pm 0.0 \mathrm{~b}$ & $6.3 \pm 5.4 a b^{1}$ & $26.6 \pm 2.4 a$ & $25.3 \pm 0.4 a$ & $0.0 \pm 0.0 \mathrm{~b}$ \\
\hline 40 & $11.3 \pm 4.5 b$ & $57.8 \pm 1.6 a$ & $72.2 \pm 1.0 \mathrm{a}$ & $0.0 \pm 0.0 c$ & $9.4 \pm 5.2 \mathrm{ab}$ & $31.4 \pm 2.9 a$ & $31.0 \pm 0.5 a$ & $0.0 \pm 0.0 b$ \\
\hline 48 & $53.0 \pm 5.1 a$ & $83.0 \pm 5.5 a$ & $85.4 \pm 3.3 a$ & $0.0 \pm 0.0 b$ & $19.0 \pm 6.1 \mathrm{ab}$ & $39.3 \pm 1.3 \mathrm{a}$ & $52.3 \pm 4.3 a$ & $0.0 \pm 0.0 \mathrm{~b}$ \\
\hline 56 & $71.2 \pm 2.9 a$ & $88.9 \pm 5.7 a$ & $93.1 \pm 2.1 \mathrm{a}$ & $0.0 \pm 0.0 b$ & $23.7 \pm 5.1 \mathrm{a}$ & $44.5 \pm 2.1 \mathrm{a}$ & $63.9 \pm 3.1 \mathrm{a}$ & $0.0 \pm 0.0 \mathrm{~b}$ \\
\hline 64 & $78.6 \pm 4.0 \mathrm{a}$ & $90.6 \pm 5.2 a$ & $93.1 \pm 2.1 \mathrm{a}$ & $0.0 \pm 0.0 b$ & $23.7 \pm 5.1 b$ & $47.2 \pm 1.8 \mathrm{ab}$ & $66.7 \pm 2.7 a$ & $0.0 \pm 0.0 \mathrm{~b}$ \\
\hline 72 & $90.4 \pm 4.0 \mathrm{a}$ & $98.7 \pm 1.6 \mathrm{a}$ & $96.1 \pm 2.1 \mathrm{a}$ & $0.0 \pm 0.0 \mathrm{~b}$ & $33.9 \pm 3.7 a$ & $50.0 \pm 1.3 a$ & $66.7 \pm 2.7 a$ & $0.0 \pm 0.0 \mathrm{~b}$ \\
\hline 80 & $90.4 \pm 4.0 \mathrm{a}$ & $98.7 \pm 1.6 a$ & $96.1 \pm 2.1 \mathrm{a}$ & $0.0 \pm 0.0 b$ & $42.0 \pm 2.0 \mathrm{a}$ & $50.0 \pm 1.3 \mathrm{a}$ & $66.7 \pm 2.7 a$ & $0.0 \pm 0.0 \mathrm{~b}$ \\
\hline 88 & $90.4 \pm 4.0 \mathrm{a}$ & $98.7 \pm 1.6 \mathrm{a}$ & $98.3 \pm 1.8 \mathrm{a}$ & $0.0 \pm 0.0 b$ & $47.8 \pm 4.7 a$ & $50.0 \pm 1.3 a$ & $69.6 \pm 2.8 a$ & $0.0 \pm 0.0 \mathrm{~b}$ \\
\hline 96 & $92.0 \pm 3.5 a$ & $98.7 \pm 1.6 a$ & $98.3 \pm 1.8 \mathrm{a}$ & $0.0 \pm 0.0 b$ & $53.3 \pm 4.6 a$ & $55.3 \pm 0.8 a$ & $80.2 \pm 1.8 a$ & $0.0 \pm 0.0 \mathrm{~b}$ \\
\hline 104 & $92.0 \pm 3.5 a$ & $98.7 \pm 1.6 \mathrm{a}$ & $98.3 \pm 1.8 \mathrm{a}$ & $0.0 \pm 0.0 b$ & $66.1 \pm 3.7 \mathrm{a}$ & $63.7 \pm 3.1 \mathrm{a}$ & $85.2 \pm 2.8 \mathrm{a}$ & $0.0 \pm 0.0 \mathrm{~b}$ \\
\hline 112 & $92.0 \pm 3.5 a$ & $98.7 \pm 1.6 \mathrm{a}$ & $98.3 \pm 1.8 \mathrm{a}$ & $0.0 \pm 0.0 b$ & $66.1 \pm 3.7 a$ & $66.1 \pm 2.8 \mathrm{a}$ & $89.6 \pm 3.5 a$ & $0.0 \pm 0.0 \mathrm{~b}$ \\
\hline \multirow[t]{3}{*}{120} & $93.4 \pm 2.8 \mathrm{a}$ & $98.7 \pm 1.6 \mathrm{a}$ & $98.3 \pm 1.8 a$ & $0.0 \pm 0.0 b$ & $66.1 \pm 3.7 a$ & $66.1 \pm 2.8 \mathrm{a}$ & $89.6 \pm 3.5 a$ & $0.0 \pm 0.0 \mathrm{~b}$ \\
\hline & \multicolumn{8}{|c|}{ S. carpocapsae Tokat-Ulas } \\
\hline & $500 \mathrm{IJs}$ & & 1000 & & 2000 & & Control & \\
\hline 32 & $16.9 \pm 10.2 \mathrm{ab}$ & & $26.1 \pm 5.17 a b$ & & $52.8 \pm 8.2 a$ & & $0.0 \pm 0.0 \mathrm{~b}$ & \\
\hline 40 & $36.8 \pm 6.8 a$ & & $33.4 \pm 5.54 a$ & & $76.3 \pm 4.2 \mathrm{a}$ & & $0.0 \pm 0.0 \mathrm{~b}$ & \\
\hline 48 & $61.0 \pm 9.9 a$ & & $76.3 \pm 4.21 a$ & & $88.7 \pm 3.5 a$ & & $0.0 \pm 0.0 \mathrm{~b}$ & \\
\hline 56 & $83.4 \pm 7.2 \mathrm{a}$ & & $88.6 \pm 3.09 a$ & & $93.4 \pm 2.8 a$ & & $0.0 \pm 0.0 \mathrm{~b}$ & \\
\hline 64 & $85.4 \pm 6.9 a$ & & $91.8 \pm 2.00 \mathrm{a}$ & & $93.4 \pm 2.8 a$ & & $0.0 \pm 0.0 \mathrm{~b}$ & \\
\hline 72 & $85.4 \pm 6.9 a$ & & $97.0 \pm 2.00 \mathrm{a}$ & & $97.0 \pm 2.0 \mathrm{a}$ & & $0.0 \pm 0.0 \mathrm{~b}$ & \\
\hline 80 & $90.6 \pm 5.2 \mathrm{a}$ & & $98.7 \pm 1.60 \mathrm{a}$ & & $98.7 \pm 1.6 a$ & & $0.0 \pm 0.0 \mathrm{~b}$ & \\
\hline 88 & $92.3 \pm 6.1 a$ & & $98.7 \pm 1.60 \mathrm{a}$ & & $98.7 \pm 1.6 a$ & & $0.0 \pm 0.0 \mathrm{~b}$ & \\
\hline 96 & $93.7 \pm 5.5 a$ & & $100.0 \pm 0.0 \mathrm{a}$ & & $100.0 \pm 0.0 \mathrm{a}$ & & $0.0 \pm 0.0 b$ & \\
\hline 104 & $95.0 \pm 3.9 a$ & & $100.0 \pm 0.0 a$ & & $100.0 \pm 0.0 \mathrm{a}$ & & $0.0 \pm 0.0 \mathrm{~b}$ & \\
\hline 112 & $95.0 \pm 3.9 a$ & & $100.0 \pm 0.0 \mathrm{a}$ & & $100.0 \pm 0.0 \mathrm{a}$ & & $0.0 \pm 0.0 \mathrm{~b}$ & \\
\hline 120 & $95.0 \pm 3.9 a$ & & $100.0 \pm 0.0 a$ & & $100.0 \pm 0.0 \mathrm{a}$ & & $0.0 \pm 0.0 b$ & \\
\hline
\end{tabular}

*SEM: standard error of the mean

**HAT: hours after treatment

***Means in a line followed by the same letter are not statistical significantly different (ANOVA $P<0.05$, Tukey's test)

bacteria within the nematodes affect its virulence. The other possibility of variation in pathogenicity of EPN could be the host finding and penetration capability of nematodes. $H$. bacteriophora (isolate 09-43) and $H$. bacteriophora Tokat-Songut showed 98 and 100\% effect on G. fornicata at $2000 \mathrm{IJs} \mathrm{ml}^{-1}$ doses in $120 \mathrm{~h}$, respectively, while this effect was determined as 26 and $37 \%$ for $H$. postica at the same time and dose. Difference in the susceptibility of host insect species may be related with their immune system and the physical structure.

Kim et al. (2007) investigated the efficiency of 4 Korean EPNs against late larvae of alfalfa weevil and reported that S. glaseri (Steiner, 1929) Dongrae strain and Heterorhabditis sp. Gyeongsan strain were more effective against $H$. postica larva than $S$. carpocapsae GSN1 strain and $H$. bacteriophora Hamyang strain. Shah et al. (2011) stated that application of H. indica Poinar, Karunakar \& David, 1992 and S. carpocapase at the rate 1 billion IJs/acre can decrease 72 and 50\% population of alfalfa weevil, $H$. postica grubs, respectively. Falahi et al. (2011) tested S. carpocapsae against $H$. postica adults under laboratory conditions at doses of 250, 500, 1000, and $2000 \mathrm{IJs} \mathrm{ml}^{-1}$ and determined the highest effect as $97 \%$ at the dose of $2000 \mathrm{IJs} \mathrm{ml}^{-1}$ at the 72nd hour as our study.

Majić et al. (2013) showed that $H$. bacteriophora application in rate of $1000 \mathrm{IJs}$ per adult of G. fornicata 
Table 5 Mortality rates of Hypera postica adults exposed to Heterorhabditis bacteriophora (isolate 09-43) and H. bacteriophora TokatSongut and controls over $120 \mathrm{~h}$ from treatment

\begin{tabular}{|c|c|c|c|c|c|c|c|c|}
\hline \multicolumn{9}{|c|}{ Mortality $\pm \mathrm{SEM}^{*}(\%)$} \\
\hline \multirow[t]{2}{*}{ HAT** } & \multicolumn{4}{|c|}{ Heterorhabditis bacteriophora (isolate 09-43) } & \multicolumn{4}{|c|}{ H. bacteriophora Tokat-Songut } \\
\hline & $500 \mathrm{IJs}$ & 1000 & 2000 & Control & $500 \mathrm{IJs}$ & 1000 & 2000 & Control \\
\hline 32 & $0.0 \pm 0.0 a^{* * *}$ & $0.0 \pm 0.0 a$ & $0.6 \pm 1.3 a$ & $0.0 \pm 0.0 \mathrm{a}$ & $0.0 \pm 0.0$ & $0.00 \pm 0.0$ & $0.0 \pm 0.0$ & $0.0 \pm 0.0$ \\
\hline 40 & $0.0 \pm 0.0 \mathrm{a}$ & $0.0 \pm 0.0 \mathrm{a}$ & $1.3 \pm 2.7 \mathrm{a}$ & $0.0 \pm 0.0 \mathrm{a}$ & $0.0 \pm 0.0 a^{1}$ & $0.00 \pm 0.0 \mathrm{a}$ & $1.7 \pm 1.8 \mathrm{a}$ & $0.0 \pm 0.0 \mathrm{a}$ \\
\hline 48 & $0.0 \pm 0.0 \mathrm{a}$ & $0.0 \pm 0.0 \mathrm{a}$ & $1.3 \pm 2.7 \mathrm{a}$ & $0.0 \pm 0.0 \mathrm{a}$ & $0.3 \pm 0.9 a$ & $0.4 \pm 1.1 a$ & $1.7 \pm 1.8 \mathrm{a}$ & $0.0 \pm 0.0 \mathrm{a}$ \\
\hline 56 & $0.0 \pm 0.0 \mathrm{a}$ & $1.7 \pm 1.8 \mathrm{a}$ & $5.1 \pm 4.2 \mathrm{a}$ & $0.0 \pm 0.0 \mathrm{a}$ & $0.3 \pm 0.9 a b$ & $0.4 \pm 1.1 \mathrm{ab}$ & $8.5 \pm 2.9 a$ & $0.0 \pm 0.0 \mathrm{~b}$ \\
\hline 64 & $0.0 \pm 0.0 \mathrm{a}$ & $1.7 \pm 1.8 \mathrm{a}$ & $5.1 \pm 4.2 \mathrm{a}$ & $0.0 \pm 0.0 \mathrm{a}$ & $0.7 \pm 2.0 \mathrm{ab}$ & $1.7 \pm 1.8 \mathrm{ab}$ & $12.6 \pm 2.4 a$ & $0.0 \pm 0.0 b$ \\
\hline 72 & $0.0 \pm 0.0 b$ & $6.9 \pm 2.1 \mathrm{ab}$ & $9.0 \pm 4.0 a$ & $0.0 \pm 00 a b$ & $2.0 \pm 2.6 a b$ & $1.7 \pm 1.8 \mathrm{ab}$ & $12.6 \pm 2.4 a$ & $0.0 \pm 0.0 b$ \\
\hline 80 & $0.0 \pm 0.0 b$ & $6.9 \pm 2.1 \mathrm{ab}$ & $16.4 \pm 4.4 a$ & $0.0 \pm 0.0 b$ & $4.0 \pm 2.8 \mathrm{ab}$ & $6.9 \pm 2.1 \mathrm{ab}$ & $19.8 \pm 1.8 a$ & $0.0 \pm 0.0 \mathrm{~b}$ \\
\hline 88 & $3.0 \pm 2.0 \mathrm{ab}$ & $6.9 \pm 2.1 \mathrm{ab}$ & $16.4 \pm 4.4 a$ & $0.0 \pm 0.0 b$ & $4.0 \pm 2.8 a b$ & $10.6 \pm 1.8 a$ & $19.8 \pm 1.8 a$ & $0.0 \pm 0.0 b$ \\
\hline 96 & $3.0 \pm 2.0 \mathrm{ab}$ & $6.9 \pm 2.1 \mathrm{ab}$ & $22.5 \pm 3.0 a$ & $0.0 \pm 0.0 b$ & $4.0 \pm 2.8 a b$ & $12.6 \pm 2.4 a$ & $19.8 \pm 1.8 a$ & $0.0 \pm 0.0 b$ \\
\hline 104 & $8.0 \pm 3.5 \mathrm{ab}$ & $10.6 \pm 1.8 a b$ & $22.5 \pm 3.0 \mathrm{a}$ & $0.0 \pm 0.0 b$ & $6.6 \pm 2.8 b c$ & $17.3 \pm 1.5 \mathrm{ab}$ & $30.7 \pm 1.0 a$ & $0.0 \pm 0.0 c$ \\
\hline 112 & $8.0 \pm 3.5 \mathrm{ab}$ & $17.3 \pm 1.5 \mathrm{a}$ & $22.5 \pm 3.0 \mathrm{a}$ & $0.0 \pm 0.0 b$ & $7.9 \pm 3.8 b c$ & $17.3 \pm 1.5 \mathrm{ab}$ & $33.6 \pm 0.9 a$ & $0.0 \pm 0.0 c$ \\
\hline 120 & $11.4 \pm 3.1 a$ & $22.6 \pm 0.3 a$ & $25.6 \pm 3.2 a$ & $0.0 \pm 0.0 \mathrm{~b}$ & $13.1 \pm 4.8 \mathrm{a}$ & $17.3 \pm 1.5 a$ & $36.7 \pm 0.8 a$ & $0.0 \pm 0.0 b$ \\
\hline
\end{tabular}

*SEM: standard error of the mean

**HAT: hours after treatment

***Means in a line followed by the same letter are not statistical significantly different (ANOVA $P<0.05$, Tukey's test)

caused $100 \%$ mortality rate on 3 day post-treatment in the laboratory. Similarly, in the present study, H. bacteriophora isolates had a significant effect on adult of $G$. fornicata. On other chrysomelids, mortality studies have been carried out with success using a variety of steinernematid and heterorhabditid. Trdan et al. (2008) assessed virulence of EPNs S. feltiae, S. carpocapsae, $H$. bacteriophora, and $H$. megidis against adult flea beetles, Phyllotreta spp. (Coleoptera: Chrysomelidae) under laboratory conditions and revealed that S. feltiae was the most effective nematode. Also, Trdan et al. (2009) stated that S. feltiae showed the highest efficacy in controlling adults of Leptinotarsa decemlineata (Say, 1824) (Coleoptera: Chrysomelidae) at $15^{\circ} \mathrm{C}$. Similarly, in the present study, S. feltiae (isolate 09-31) was more effective on G. fornicata than $H$. postica. Unlike these studies, Laznik et al. (2010) determined that S. carpocapsae $\mathrm{C} 101$ caused more mortality than S. feltiae B30 and $H$. bacteriophora D54 against Oulema melanopus (Linnaeus, 1758) (Coleoptera: Chrysomelidae) adults.

\section{Conclusions}

This study highlighted the first analysis of EPNs' efficacy against H. postica and G. fornicata in Turkey. G. fornicata adults were susceptible to all isolates tested, while $H$. postica adults were susceptible to $S$. carpocapsae (Black Sea isolate), S. feltiae (isolate 09-31), and S. carpocapsae Tokat-Ulas isolates. Therefore, these native EPNs can be used as bio-control agents against the adults of these 2 important alfalfa pests. Further studies should be conducted under field conditions.

\section{Abbreviations}

EPNs: Entomopathogenic nematodes; IJs: Infective juveniles; LT: Lethal time

Table 6 Lethal time ( $L T_{30}, L_{50}$, and $\left.L T_{90}\right)$ values of treated isolates against adults of Hypera postica (hour)

\begin{tabular}{|c|c|c|c|c|c|}
\hline Isolates & Slope \pm SE & $\mathrm{LT}_{30}(95 \%$ fiducial limit $)$ & $\mathrm{LT}_{50}(95 \%$ fiducial limit $)$ & $\mathrm{LT}_{90}(95 \%$ fiducial limit $)$ & Heterogeneity \\
\hline $\begin{array}{l}\text { Steinernema carpocapsae } \\
\text { (Black sea isolate) }\end{array}$ & $3.869 \pm 0.283$ & $25.916(22.380-29.113)$ & 35.407 (31.790-38.848) & $75.909(68.501-86.031)$ & 1.14 \\
\hline S. feltiae (isolate 09-31) & $2.381 \pm 0.249$ & $45.810(39.570-51.478)$ & $76.074(68.288-85.999)$ & 262.756 (202.900-384.894) & 0.77 \\
\hline $\begin{array}{l}\text { Heterorhabditis bacteriophora } \\
\text { (isolate 09-43) }\end{array}$ & $4.424 \pm 0.809$ & 121.745 (109.212-147.749) & 159.954 (135.437-222.873) & 311.667 (223.473-624.159) & 0.43 \\
\hline H. bacteriophora Tokat-Songut & $4.341 \pm 0.683$ & 108.901 (99.625-124.271) & 143.823 (125.686-182.167) & 283.818 (213.812-481.143) & 0.87 \\
\hline S. carpocapsae Tokat-Ulas & $5.073 \pm 0.376$ & $29.840(26.880-32.501)$ & $37.858(34.967-40.629)$ & $67.729(62.496-74.601)$ & 0.95 \\
\hline
\end{tabular}




\section{Acknowledgements}

The authors thank to Prof. Dr. Selçuk HAZIR (Aydın Adnan Menderes University, Faculty of Arts and Sciences, Department of Biology, Aydın, Turkey) for providing some entomopathogenic nematode isolates.

\section{Authors' contributions}

AÇ, TA, and IK conceived and designed the research. AÇ conducted the experiments. TA analyzed the data and wrote the manuscript. IK corrected and revised the manuscript, corrected language mistakes and translation, and corrected references. All authors read and approved the final manuscript.

\section{Funding}

The study was supported by the Tokat Gaziosmanpaşa University Scientific Research Fund (project number: 2017/55).

\section{Availability of data and materials}

The dataset(s) supporting the conclusions of this article is (are) included within the article (and its additional file(s)).

\section{Declarations}

\section{Ethics approval and consent to participate}

Not applicable

\section{Consent for publication}

Not applicable

\section{Competing interests}

The authors declare that they have no competing interests.

Received: 22 March 2021 Accepted: 30 May 2021

Published online: 08 June 2021

\section{References}

Atanasova DY, Semerdjieva IB (2009) Populatıon density of Phytonomus variabilis Hrbst. and Phytodecta fornicata Brugg. on multifoliolate and trifoliolate alfalfa in relation to anatomical characteristics on their leaves. J Central Eur Agric 10(4):321-326

Atay T, Kepenekci I (2016) Biological control potential of Turkish entomopathogenic nematodes against Holotrichapion pullum (Gyllenhal) (Coleoptera, Apionidae). Egypt J Biol Pest Control 26:7-10

Belien T (2018) Entomopathogenic nematodes as biocontrol agents of insect pests in orchards. CAB Rev. 13(058):1-11. https://doi.org/10.1079/PAVSNNR2 01813058

Brivio MF, Mastore M (2018) Nematobacterial complexes and insect hosts: different weapons for the same war. Insects 9(117):2-30. https://doi.org/10.33 90/insects9030117

Çağlayan A, Atay T, Kepenekci I (2020) Entomopathogenic nematodes occurring in alfalfa fields, Tokat, Turkey. Plant Protection Bulletin 60(4):41-47. https:// doi.org/10.16955/bitkorb.749288

Efe D, Özgökçe S (2014) The life table of the lucerne beetle, Gonioctena fornicata (Brüggem) (=Phytodecta fornicatus Brüggem) (Coleoptera, Chrysomelidae) on alfalfa under laboratory conditions. Türk. entomol. derg. 38(1):3-10

Efil (2018) The damage status of Alfalfa Weevil Hypera variabilis (Herbst, 1795) (Coleoptera: Curculionidae) in the alfalfa areas of Diyarbakır, Şanlıurfa, Mardin provinces and parasitoid Bathyplectes curculionis (Thomson, 1887) and parazitization. Türk Tarım ve Doğa Bilimleri Dergisi 5(1):86-89

Falahi M, Abdollahi M, Roodaki M, Haghani M (2011) Efficacy of Steinernema carpocapsae for control of the adults of alfalfa weevil, Hypera postica. Paper presented at the National Conference on Modern Agricultural Sciences and Technologies (MAST). Zanjan University, Zanjan

Glazer I, Lewis EE (2000) Bioassays for entomopathogenic nematodes. In: Navon A, Ascher KRS (eds) Bioassays of entomopathogenic microbes and nematodes. CAB International, pp 229-247

Gözüaçık C, Ireç A (2019) Some biological aspects of alfalfa weevil, Hypera postica (Gyllenhal, 1813) (Coleoptera: Curculionidae) J. Inst. Sci. Technol. 9:1220-1225. https://doi.org/10.21597/jist.515642

Gulcu B, Cimen H, Raja RK, Hazir S (2017) Entomopathogenic nematodes and their mutualistic bacteria: their ecology and application as microbial control agents. Biopestic. Int 3(2):79-112
Karakurt E, Fırıncıoğlu HK (2003) Farklı kaynaklardan sağlanan Yonca (Medicago sativa L.) populasyonunda bazı önemli özellikler ve özellikler arası ilişkiler. Tarla Bitkileri Merkez Araştırma Enstitüsü (TARM) Dergisi 12(1-2):86-94

Kaya HK, Stock SP (1997) Techniques in Insect Nematology. In: Lacey LA (ed) Manual of techniques in insect pathology. Academic press, London, pp $281-$ 324. https://doi.org/10.1016/B978-012432555-5/50016-6

Kepenekci I, Toksöz Ş, Atay T, Saruhan I (2018) Efficacy of entomopathogenic nematode isolates from Turkey and Kyrgyzstan against Black Timber Bark Beetle, Xylosandrus germanus (Blandford) (Coleoptera: Curculionidae: Scolytinae) Adults. Pak. J. Nematol. 36(1):65-70. https://doi.org/10.18681/pjn. v36.101.p65-70

Kim HH, Han GY, Park CC, Choo HY, Cho SR, Lee HS, Lee DW, Park CG (2007) Susceptibility of the Alfalfa Weevil, Hypera postica (Coleoptera: Curculionidae) to Korean entomopathogenic nematodes in laboratory assays. Korean J. Appl. Entomol. 46(1):147-151. https://doi.org/10.5656/KSAE.2007.46.1.147

Laznik Ž, Tóth T, Lakatos T, Vidrih M, Trdan S (2010) Oulema melanopus (L.) (Coleoptera: Chrysomelidae) adults are susceptible to entomopathogenic nematodes (Rhabditida) attack: results from a laboratory study. J. Plant Dis. Protect 117(1):30-32

Majić I, Raspudić E, Ivezić M, Brmež M, Sarajlić A, Mirković A (2013) Susceptibility of Phytodecta fornicata (Coleoptera: Chrysomelidae) to Heterorhabditis bacteriophora. Insect Pathogens Entomoparasitic Nematodes 90:309-312

Odendaal D, Addison MF, Antoinette P (2015) Entomopathogenic nematodes for the control of the codling moth (Cydia pomonella L.) in field and laboratory trials. J Helminthol 1(5):1-9

Roodaki M, Haghani M, Falahi M, Abdollahi M (2011) The response of the alfalfa weevil, Hypera postica, adults to Steinernema feltiae in vitro. Paper presented at the National Conference on Modern Agricultural Sciences and Technologies (MAST). Zanjan University, Zanjan

Shah NK, Azmi MI, Tyagi PK (2011) Pathogenicity of Rhabditid nematodes (Nematoda: Heterorhabditidae and Steinernematidae) to the grubs of alfalfa weevil, Hypera postica (Coleoptera: Curculionidae). Range Management and Agroforestry 32:64-67

Shahina F, Salma J (2010) Laboratory evaluation of seven Pakistani strains of entomopathogenic nematode against stored grain insect pest Sitophilus oryzae L. Pak. J. Nematol. 28(2):295-305

Trdan S, Vidrih M, Anjus L, Laznik Z (2009) Activity of four entomopathogenic nematode species against different developmental stages of Colorado potato beetle, Leptinotarsa decemlineata (Coleoptera, Chrysomelidae). Helminthologia 46(1):14-20. https://doi.org/10.2478/s11687-009-0003-1

Trdan S, Vidrih M, Valic N, Laznik Z (2008) Impact of entomopathogenic nematodes on adults of Phyllotreta spp. (Coleoptera: Chrysomelidae) under laboratory conditions. Acta Agric Scand B Soil Plant Sci 58:169-175

White GF (1927) A method for obtaining infective nematode larvae from cultures. Science 66(1709):302-303. https://doi.org/10.1126/science.66.1 709.302-a

\section{Publisher's Note}

Springer Nature remains neutral with regard to jurisdictional claims in published maps and institutional affiliations.

\section{Submit your manuscript to a SpringerOpen ${ }^{\circ}$ journal and benefit from:}

- Convenient online submission

- Rigorous peer review

- Open access: articles freely available online

- High visibility within the field

- Retaining the copyright to your article

Submit your next manuscript at $>$ springeropen.com 